\title{
3D Ray Tracing for Device-Independent Fingerprint-based Positioning in WLANs
}

\author{
Marios Raspopoulos*, Christos Laoudias ${ }^{\dagger}$, Loizos Kanaris*, Akis Kokkinis*, Christos G. Panayiotou ${ }^{\dagger}$, Stavros Stavrou $^{\ddagger}$ \\ * Sigint Solutions Ltd, Nicosia, Cyprus \\ Email: \{m.raspopoulos, 1.kanaris, a.kokkinis\}@sigintsolutions.com \\ $\dagger$ KIOS Research Center for Intelligent Systems and Networks \\ Department of Electrical and Computer Engineering, University of Cyprus \\ Email:\{laoudias, christosp\}@ucy.ac.cy \\ ${ }_{\ddagger}$ Faculty of Pure and Applied Sciences, Open University of Cyprus \\ Email:stavros.stavrou@ouc.ac.cy
}

\begin{abstract}
We study the use of 3D Ray Tracing (RT) to construct radiomaps for WLAN Received Signal Strength (RSS) fingerprint-based positioning, in conjunction with calibration techniques to make the overall process device-independent. RSS data collection might be a tedious and time-consuming process and also the measured radiomap accuracy and applicability is subject to potential changes in the wireless environment. Therefore, RT becomes a more attractive and efficient way to generate radiomaps. Moreover, traditional fingerprint-based methods lead to radiomaps which are restricted to the device used to generate the radiomap and fail to provide acceptable performance when different devices are considered. We address both challenges by exploiting 3D RT-generated radiomaps and using linear data transformation to match the characteristics of various devices. We evaluate the efficiency of this approach in terms of the time spent to create the radiomap, the amount of data required to calibrate the radiomap for different devices and the positioning error which is compared against the case of using dedicated radiomaps collected with each device.
\end{abstract}

\section{INTRODUCTION}

Fingerprint-based positioning became a very popular topic of research in the communication research community over the last decades [1]. It consists of two main phases; the offline phase where pre-measured location-dependent signal information (e.g. RSS), known as "fingerprints" that cover the entire area of interest, are stored in the database (radiomap) and an online phase where the instantaneous measurement is correlated with the fingerprints in the radiomap to estimate the position.

Generating and maintaining the radiomap is very important and it can be either done through an extensive measurement campaign or through radio propagation modeling techniques. Experimental measurements might lead to more accurate fingerprints, but this process might be very laborious and also the applicability of the measured radiomap is reduced if the wireless environment is changed. Therefore, propagation modeling techniques, such as Ray Tracing, have been used for the creation and maintenance of the radiomap [2]-[5]. Still, RT accuracy is subject to the precise definition of the geometry and morphology (e.g., wall electrical parameters) of the environment and also the accurate definition of the transmitter and receiver antennas. Such information is usually hard to obtain and this might necessitate the crude calibration of the RT tool in order to achieve higher accuracy.

Another basic limitation of fingerprint-based techniques is that the device heterogeneity may degrade the positioning performance when the device to be positioned is different from the device that was used to collect the radiomap. Differences may arise due to the different antenna characteristics of the mobile terminals which are usually difficult to know or predict. There is work reported in literature that tries to address the issue of device diversity; mainly by calibrating the RSS measurements collected from a mobile device to be positioned to match the fingerprints contained in the radiomap (created using another device), either through linearly transforming the collected measurements with each device to match the fingerprints in the radio map [6], [7], or by recording the signal ratios between pairs of APs [8]. In this work we address both challenges by using a single artificial radiomap generated through 3D RT simulations and thereafter use linear transformation to fit this radiomap to a set of fingerprints collected using four different WLAN-enabled devices. Specifically, we focus on the amount of fingerprints that need to be collected to obtain appropriate linear transformation parameters that guarantee low positioning error for each device.

Section II describes the fingerprint radiomap generation using both measurements and RT simulations, Section III describes the device calibration procedure which is applied on the simulated and measured data. Section IV summarises the results and performance of our approach. Finally, Section V provides concluding remarks and ideas for future work.

\section{FINGERPRINT RADIOMAP GENERATION}

In order to assess the use of RT-generated radiomaps for enabling fingerprint-based positioning with diverse devices, WLAN RSS measurements have been collected in an indoor wireless environment which has been also modeled and simulated using a 3D RT Simulator.

For the measurements, we have used 3 Android-based handsets (HTC Desire HD, Samsung Nexus S and Samsung 
Galaxy Tab) and one laptop (Lenovo X100e). Measurements have been performed at the same time with all four devices logging data from up to 6 D-Link $802.11 \mathrm{~b}$ APs installed inside the building and the RSS values range from $-98 \mathrm{dBm}$ to $-15 \mathrm{dBm}$. Data was measured at 110 equally-spaced $(1 \mathrm{~m}$ spacing) training locations. At every location 30 fingerprints have been recorded (1 sample/sec) and the mean fingerprint value, averaged for each AP, has been computed to build each device-specific radiomap. The whole data collection process took 2 hours to complete. For testing purposes additional RSS fingerprints have been collected with all devices along a route that comprises 40 distinct locations, while 10 fingerprints were measured at every test location with no averaging. The floorplan of the experimentation area, the installed APs, the training locations and test route are depicted in Figure 1. To enable random selection of data, distributed uniformly in the environment, we divide the whole area into seven nonoverlapping regions $A_{i}, i=1, \ldots, 7$ representing rooms and large open spaces (see Figure 1), i.e. $\left\{A_{1}: \ell_{j}, j=1, \ldots, 11\right\}$, $\left\{A_{2}: \ell_{j}, j=12, \ldots, 30\right\},\left\{A_{3}: \ell_{j}, j=31, \ldots, 40\right\}$, $\left\{A_{4}: \ell_{j}, j=41, \ldots, 59\right\},\left\{A_{5}: \ell_{j}=60, \ldots, 69\right\}$, $\left\{A_{6}: \ell_{j}, j=70, \ldots, 89\right\},\left\{A_{7}: \ell_{j}, j=90, \ldots, 110\right\}$ where $j$ is the training location index.

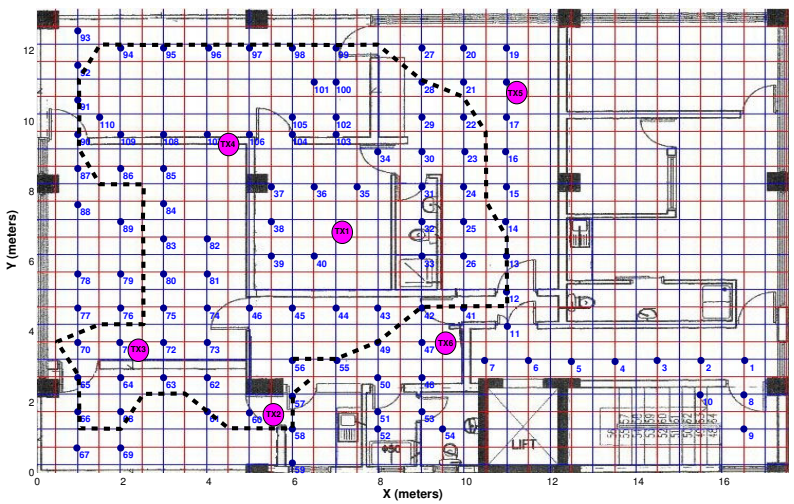

Fig. 1. Experimentation Area Floor Plan (Reference Locations and APs).

Also, the radiomap has been created using 3DTruEM; a powerful 3D Ray Tracing Simulator developed by Sigint Solutions Ltd. Its calculation engine relies on a RT algorithm which uses the 3D EM formulation of reflection, refraction and diffraction based on the Universal Theory of Diffraction (UTD). It offers the ability to define the receiver and transmitter antenna characteristics from a wide range of standard antennas, as well as the flexibility to import a custom-made antenna by defining its 3D radiation pattern. A snapshot of our simulator user interface is shown in Figure 2.

The indoor environment has been modeled into the RT Simulator by importing its CAD file into the CAD designer including features of the environment such as desks, tables etc. The 110 receiving locations have been defined at the same positions where the measurements with the devices have been carried out. In every receiving location a rectangular grid of 36 equally-spaced $(10 \mathrm{~cm}$ ) isotropic receivers (at $90 \mathrm{~cm}$ height) have been defined in order to remove potential fast fading behavior by obtaining their local average. The accuracy of the RT simulator relies strongly on the precise definition of the electrical parameters of the walls defined in the geometric model of the environment and also on the accurate antenna pattern used. For this reason a Ray Tracing calibration process [9] was carried out in order to fine-tune the walls constitutive parameters and improve the accuracy of the RT simulations when these are compared with the measurements received using the Nexus device. Some of the calibration iterations are tabulated in Table I. As a first iteration, random electrical parameters have been used to characterise the walls and also for the 6 access points (at $2.3 \mathrm{~m}$ height) we have used antenna patterns for typical dipole antennas. This iteration has achieved an error of $7.2 \pm 5.22 \mathrm{~dB}$. In addition to the fact that the parameters used were unrealistic, the presence of the wall in the near-field of the antenna slightly modifies its pattern. For this reason we have carried out in-situ measurements using a spectrum analyser to better characterise the AP antenna pattern. We have used this pattern in the second calibration iteration together with typical electrical parameters obtained from literature [10] decreasing the error to $6.77 \pm 4.29 d B$. At the final iteration (11) the parameters have been fine-tuned, further decreasing the error to $5.62 \pm 4.23 \mathrm{~dB}$. This error is mainly due to the fact that the receiver antenna pattern is unknown and it has been assumed as isotropic. To investigate the effect of diffraction an additional iteration was carried out showing minor effect on the accuracy (arround $0.5 \pm 0.2 d B$ ). Also, for positioning accuracy investigations (see later) the COST model was also used to create the radio map (error $13.1 \pm 23.6 \mathrm{~dB})$. The radiomaps generated at these iteration steps will be used in section III to investigate the effect of the RT calibration on the positioning accuracy.

TABLE I

RAY TRACING CALLIBRATION ITERATIONS

\begin{tabular}{|c|c|c|c|}
\hline Iteration & Description & $\begin{array}{c}\text { Mean } \\
\text { Error }(\mathrm{dB})\end{array}$ & $\begin{array}{l}\text { Std. } \\
\text { Deviation (dB }\end{array}$ \\
\hline 1 & $\begin{array}{l}\text { Random Parameters } \\
\text { + Dipole Antennas }\end{array}$ & 7.2 & 5.22 \\
\hline 2 & $\begin{array}{l}\text { Typical Parameters } \\
+ \text { Custom Antennas }\end{array}$ & 6.77 & 4.29 \\
\hline 11 & Fine-Tuned Parameters & 5.62 & 4.23 \\
\hline 12 & $\begin{array}{l}\text { Like iteration } 11 \\
\text { with out Diffraction }\end{array}$ & 6.04 & 4.47 \\
\hline COST & COST 231 Model & 13.1 & 23.6 \\
\hline
\end{tabular}

It is also of special interest to investigate the amount of device-specific data that are required to assist this RT calibration process and achieve acceptable accuracy. For this reason data is selected by varying the number of training locations which are used in this process. To achieve uniform distribution of the training data in the area we randomly selected a specific number of locations in every each region described earlier. Using the radiomap generated at the last calibration iteration (11) and by randomly selecting training locations in every region of the environment we compared the accuracy of the simulations against the device measurements. 


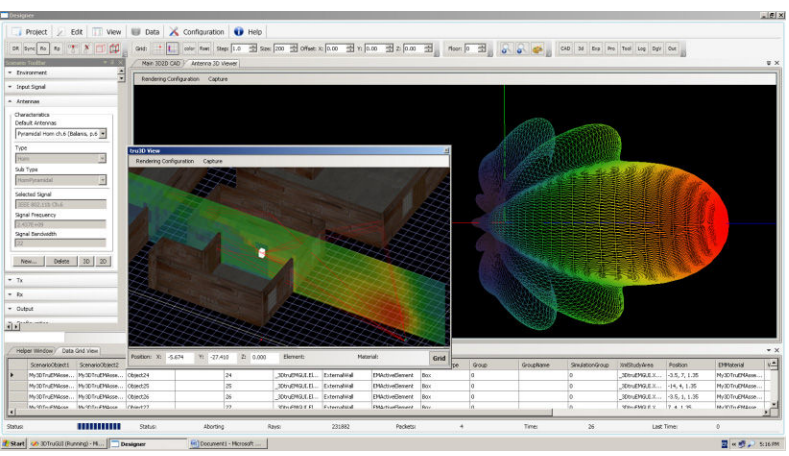

Fig. 2. 3DTruEM Ray Tracing Simulator

The result shown in Figure 3 indicates that with few samples per region the mean error and standard deviation do not vary significantly, however increasing the number of locations leads to higher confidence on these statistics. If a $\pm 0.5 \mathrm{~dB}$ confidence interval on these statistics is acceptable, 4 locations per region (i.e. 28 locations in total) are enough to achieve a good accuracy between the measurements and the simulations.
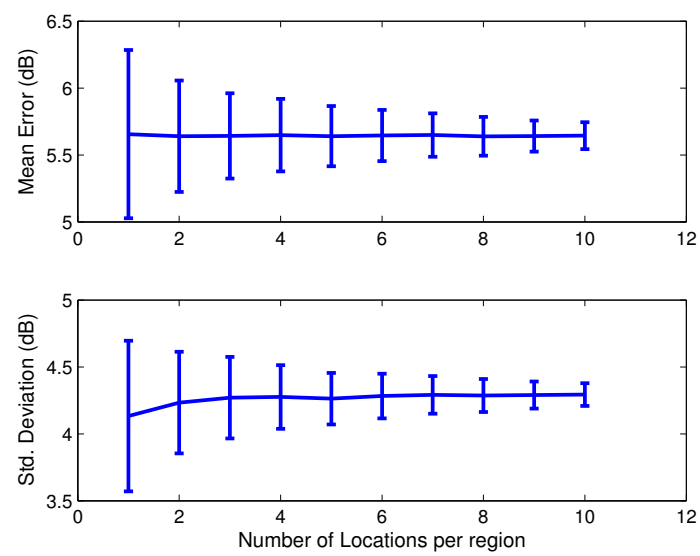

Fig. 3. Ray Tracing Calibration with Partial Measurement Data

\section{DeVice CAlibration}

The fine-tuned RT radiomap can be used thereafter as a reference radiomap for positioning various devices. However, the mobile devices report different RSS values depending on the hardware vendor of the WLAN adapter or the antenna sensitivity and pattern. Therefore, the range of RSS values can greatly vary among devices, thus rendering the direct use of a single reference radiomap questionable. This necessitates the use of device calibration to deliver a consistent level of performance, regardless of the device used during positioning.

In our approach we investigate linear transformation by using the training data for mapping the RSS values recorded with each target device to the RT radiomap. The RT radiomap contains the expected (mean) RSS value of each AP at every training location inside the area of interest. For this reason, we perform the linear data fitting in a least-squares sense using

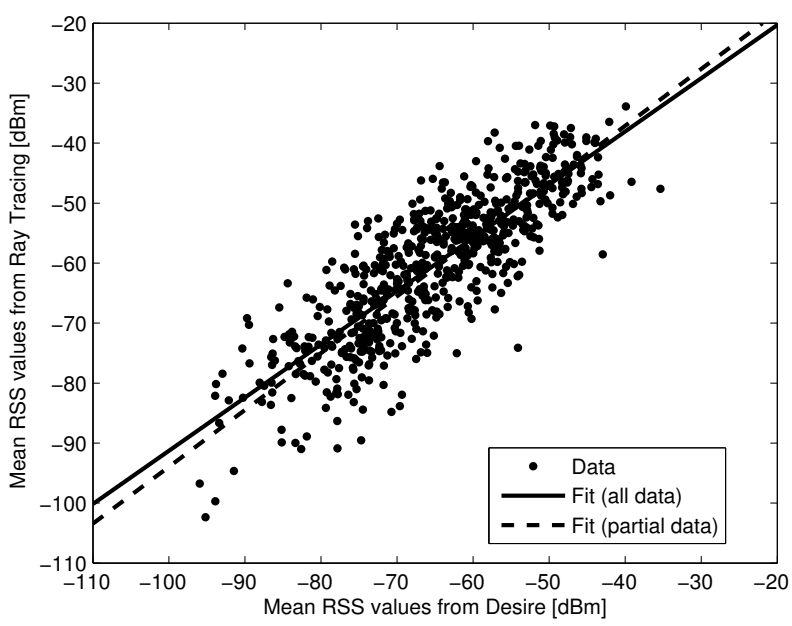

Fig. 4. Device calibration through linear fitting between the Ray Tracing radiomap and the data collected with HTC Desire.

the mean RSS values of each device averaged over multiple fingerprints collected at each training location. In this fashion, the two linear coefficients are estimated and can be used in a pre-processing step during positioning in order to scale the observed RSS values accordingly.

The data fitting between the RT radiomap and the HTC Desire is illustrated in Figure 4 indicating a strong linear correlation between the respective mean RSS values. Interestingly, the linear fitting obtained by using only $10 \%$ of the training data (dashed line), i.e. the mean RSS values in the fingerprints from 11 randomly selected locations, is very close to the respective fitting when all available training data are considered (solid line). This implies that we may use only few data for the device calibration to considerably reduce the data collection time for all target devices, thus increasing the applicability of our approach.

\section{PERformance EVAluation}

We assess the effectiveness of the proposed approach with respect to the positioning accuracy in an indoor environment and compare it with the case of using device-specific radiomaps collected with each device. Specifically, we employ the experimental data collected with all four commercial devices, as detailed in Section II, in order to investigate the positioning error pertaining to our testing dataset. We focus on the improvement achieved solely by combining the RT radiomap with the device calibration, rather than the fingerprint-based positioning method itself. Thus, our results are obtained using the well known Nearest Neighbor (NN) method [2]. Note that the proposed approach is independent of the underlying fingerprint-based method and using a more sophisticated approach, including probabilistic methods, would incur additional accuracy improvement in the overall positioning system.

First, we examine the performance in case the Ray Tracing radiomap is utilized without any device calibration. Our findings are summarized in Table II that shows the statistics 
of the positioning error, while the respective results if devicespecific radiomaps had been used are shown in parentheses for comparison. Our first observation is that for the Nexus S and Galaxy Tab devices the performance when the RT radiomap is employed for positioning is similar with the case of using device-specific radiomaps. Specifically, the mean error remains the same for the Nexus $S$, while for the Galaxy Tab it is slightly increased ( $2.2 \mathrm{~m}$ compared to $1.9 \mathrm{~m})$. However, the RT radiomap fails to provide adequate positioning accuracy for the Desire and X100e devices, without any calibration. For these two devices the mean error is increased by $0.9 \mathrm{~m}$ and $1.3 \mathrm{~m}$, respectively and this suggests that there is room for improving accuracy by means of device calibration.

TABLE II

POSITIONING ERROR [M] USING AN UNCALIBRATED RT RADIOMAP COMPARED TO DEVICE-SPECIFIC RADIOMAPS.

\begin{tabular}{lcccc}
\hline & X100e & Desire & Nexus S & Galaxy Tab \\
\hline Mean & $4.4(3.1)$ & $3.0(2.1)$ & $2.2(2.2)$ & $2.2(1.9)$ \\
Median & $4.4(2.6)$ & $2.5(1.6)$ & $2.1(2.0)$ & $2.1(1.5)$ \\
67\% cdf & $5.1(3.5)$ & $3.3(2.2)$ & $2.8(2.5)$ & $2.7(2.5)$ \\
95\% cdf & $7.6(7.1)$ & $6.7(4.4)$ & $4.9(4.7)$ & $4.7(4.6)$ \\
Max & $11.3(11.1)$ & $8.4(10.2)$ & $7.5(8.5)$ & $8.6(9.4)$ \\
\hline
\end{tabular}

The question is how much training data are required to calibrate each device and ensure a good mapping between the RSS values observed during positioning and the RT radiomap. We investigate this by employing the device-specific training data and study the effect of using part of the data for calibrating the devices, as detailed in Section III. Similarly to the RT calibration process in Section II, partial data is selected by varying the number of training locations in our setup that contribute their mean value fingerprints in the calibration process. In order to achieve a uniform distribution of the training data utilized for calibration we randomly select a specific number of locations from each region described in Section II. The average and standard deviation of the mean and $95 \%$ CDF positioning error, obtained over 100 runs using a variable number of randomly selected locations per region in each run, are tabulated in Table III. In this case all 30 fingerprints available for each location are used to calculate the mean RSS fingerprints for the device calibration.

TABLE III

POSITIONING ERROR [M] USING A VARIABLE NUMBER OF TRAINING LOCATIONS PER REGION.

\begin{tabular}{lcccc}
\hline & X100e & Desire & Nexus S & Galaxy Tab \\
\hline \multicolumn{5}{c}{ Mean error } \\
1 location & $3.3 \pm 0.1$ & $2.4 \pm 0.1$ & $2.3 \pm 0.1$ & $2.5 \pm 0.2$ \\
4 locations & $3.3 \pm 0.0$ & $2.4 \pm 0.0$ & $2.3 \pm 0.0$ & $2.5 \pm 0.1$ \\
All locations & 3.3 & 2.4 & 2.3 & 2.4 \\
\hline \hline & \multicolumn{5}{c}{$\mathbf{9 5 \%}$ CDF error } \\
1 location & $7.9 \pm 0.4$ & $5.6 \pm 0.7$ & $4.9 \pm 0.3$ & $5.9 \pm 0.8$ \\
4 locations & $8.0 \pm 0.1$ & $5.8 \pm 0.3$ & $5.0 \pm 0.1$ & $5.9 \pm 0.3$ \\
All locations & 8.0 & 5.9 & 4.9 & 5.8 \\
\hline
\end{tabular}

Regarding the mean positioning error, shown in rows 13 , it seems that using the data from only one location per
TABLE IV

PERFORMANCE OF THE PROPOSED APPROACH USING 4 LOCATIONS PER REGION AND 5 FINGERPRINTS PER LOCATION FOR DEVICE CALIBRATION.

\begin{tabular}{lcccc}
\hline & X100e & Desire & Nexus S & Galaxy Tab \\
\hline Mean & $3.4 \pm 0.0$ & $2.4 \pm 0.0$ & $2.3 \pm 0.0$ & $2.5 \pm 0.1$ \\
Median & $2.7 \pm 0.1$ & $2.1 \pm 0.0$ & $2.1 \pm 0.0$ & $2.1 \pm 0.1$ \\
67\% cdf & $3.6 \pm 0.1$ & $2.5 \pm 0.0$ & $2.8 \pm 0.0$ & $2.8 \pm 0.1$ \\
95\% cdf & $8.0 \pm 0.0$ & $5.8 \pm 0.3$ & $5.0 \pm 0.1$ & $5.9 \pm 0.2$ \\
Max & $10.4 \pm 0.4$ & $9.0 \pm 0.3$ & $7.5 \pm 0.1$ & $8.5 \pm 0.2$ \\
\hline
\end{tabular}

region, provides the same performance with the case when the data from all 110 training locations are used. The standard deviation is also very low, indicating that the mean error is not affected by the selection of specific locations in each region. On the other hand, data from more locations per region should be used to narrow the confidence interval for the 95\% CDF positioning error (rows 4-6). For instance, using data from four locations per region decreases further the uncertainty around the expected value of the $95 \% \mathrm{CDF}$ error. This is justified because these partial data actually contain several RSS values from all APs, which cover a wide range of values, thus the data fitting during the device calibration is very effective.

Another important issue is the number of fingerprints, containing raw RSS values, that need to be collected at each location in order to calculate the mean RSS fingerprints, as it can greatly affect the time spent at a particular location for collecting a series of samples. Thus, we additionally varied the number of fingerprints that contribute to the mean RSS fingerprint at each location. Our preliminary results suggest that by using only five fingerprints per location does not affect the performance of the proposed approach and provides the same positioning accuracy, as with the case of using all 30 fingerprints per location. Combining this with our previous finding on the number of training locations leads to great time savings with respect to the device calibration process, as only a small fraction of time needs to be spent for collecting devicespecific data.

The accuracy results, assuming data from only four locations per region and five fingerprints per location are used in the device calibration, are reported in Table IV. For the $\mathrm{X} 100 \mathrm{e}$ and Desire devices the proposed approach improves considerably the performance compared to using the RT radiomap without any calibration, while the positioning error is close to the error achieved when device-specific radiomaps are employed; see Table II for comparison. For the Nexus S device similar behavior was observed, while in the case of Galaxy Tab the device calibration leads to some higher errors during positioning that slightly increase the mean error.

In any case, the traditional fingerprint-based approach can be replaced by the proposed approach, which requires only five fingerprints at four random locations in each of the seven regions inside our experimentation area for device calibration. The amount of fingerprints collected can be also used in order to fine-tune the electrical parameters of the building walls in order to improve the RT positioning accuracy as presented in Section II. Essentially, less than five minutes of tedious data 
TABLE V

POSITIONING ACCURACY [M] VS RT ACCURACY [DB]

\begin{tabular}{lccccc}
\hline Iteration & $\begin{array}{c}\text { Model } \\
\text { Accuracy }\end{array}$ & X100e & Desire & Nexus S & Galaxy Tab \\
\hline COST & $13.1 \pm 23.6$ & $4.3 \pm 0.1$ & $2.8 \pm 0.3$ & $3.2 \pm 0.2$ & $3.3 \pm 0.2$ \\
$\mathbf{1}$ & $7.2 \pm 5.22$ & $3.3 \pm 0.0$ & $2.7 \pm 0.0$ & $2.7 \pm 0.1$ & $2.5 \pm 0.0$ \\
$\mathbf{2}$ & $6.77 \pm 4.29$ & $3.6 \pm 0.1$ & $2.9 \pm 0.0$ & $2.9 \pm 0.1$ & $2.6 \pm 0.1$ \\
$\mathbf{1 1}$ & $5.62 \pm 4.23$ & $3.4 \pm 0.0$ & $2.4 \pm 0.0$ & $2.3 \pm 0.0$ & $2.5 \pm 0.1$ \\
$\mathbf{1 2}$ & $6.04 \pm 4.47$ & $3.4 \pm 0.1$ & $2.4 \pm 0.0$ & $2.4 \pm 0.0$ & $2.6 \pm 0.1$ \\
\hline
\end{tabular}

TABLE VI

POSITIONING ACCURACY [M] ATTAINED WITH VARIOUS ALGORITHMS

\begin{tabular}{lcccc}
\hline & X100e & Desire & Nexus S & Galaxy Tab \\
\hline KNN $(K=1)$ & $3.4 \pm 0.0$ & $2.4 \pm 0.0$ & $2.3 \pm 0.0$ & $2.5 \pm 0.1$ \\
KNN $(K=5)$ & $2.9 \pm 0.1$ & $1.9 \pm 0.0$ & $2.0 \pm 0.0$ & $1.9 \pm 0.0$ \\
WKNN $(K=5)$ & $2.9 \pm 0.1$ & $1.9 \pm 0.0$ & $2.0 \pm 0.0$ & $1.9 \pm 0.0$ \\
Probabilistic $(\sigma=6)$ & $2.9 \pm 0.0$ & $1.9 \pm 0.0$ & $2.0 \pm 0.0$ & $1.9 \pm 0.0$ \\
\hline
\end{tabular}

collection is needed for each target device, compared to around two hours of RSS data logging for building each devicespecific radiomap. If we also consider that RT simulations for this wireless environment took about 40 minutes to generate the radiomap the total time saving is around $60 \%$.

It is also interesting to investigate the effect of the RT simulation accuracy on the positioning accuracy. Table $\mathrm{V}$ shows the positioning accuracy achieved using the radiomaps obtained at the various RT calibration iterations in conjunction with the linear fitting technique described above. Compared to the COST231 model, RT leads to better positioning accuracy even if random or typical uncalibrated parameters are used for characterising the building walls. If the electrical parameters are fine-tuned (iteration 11) in order to better match a set of measurement data (as presented in Section II) the positioning accuracy is significantly improved. Also, ignoring diffraction has minimal effect in the RT simulation accuracy and effectively on the positioning precision; compare the results obtained at the $11^{\text {th }}$ and $12^{\text {th }}$ iteration in Table V. This opposes to the findings in [11] where it is claimed that ignoring the effect of diffraction leads to $700 \%$ degradation in the positioning accuracy.

As mentioned earlier, the proposed approach is independent of the underlying fingerprinting method and more sophisticated methods are expected to incur additional accuracy improvement in the overall positioning system. This is verified in Table VI which tabulates the mean positioning error of several positioning techniques; the KNN method [2] with $K=5$, the Weighted KNN method [12] with $K=5$ and a probabilistic technique based on the Bayesian Inference method with kernel width $\sigma=6$ [13].

\section{CONClusion}

Fingerprint-based positioning with respect to device diversity is an active research field because the time consuming data collection process, using several target devices, is involved in the construction of the necessary radiomap. In this work we focus on the use of a powerful 3D RT simulator to automatically obtain a reference radiomap with much less effort. Subsequently, we combine that with a device calibration phase, which is based on linear fitting, to effectively map the RSS values observed during positioning to our reference RT radiomap, irrespectively of the user device. The proposed approach mitigates the cumbersome task of recording large datasets of RSS values throughout the area of interest with multiple devices. Our performance evaluation indicates that only a small amount of device-specific data are required to reach the same level of positioning accuracy attained with a manually collected radiomap. Thus, our approach is far less laborious compared to traditional radiomap construction. Moreover, the radiomap can be easily updated if the propagation environment changes in the future (e.g. APs are added or removed, furniture or heavy equipment is relocated, etc.) by running the RT simulator, instead of collecting the radiomap data from scratch.

\section{ACKNOWLEDGMENT}

This work has been performed in the framework of the ICT248894 WHERE2 project funded by the European Union.

\section{REFERENCES}

[1] M. Kjærgaard, "A taxonomy for radio location fingerprinting," in 3rd international conference on Location-and context-awareness. SpringerVerlag, 2007, pp. 139-156.

[2] P. Bahl and V. Padmanabhan, "RADAR: an in-building RF-based user location and tracking system," in IEEE International Conference on Computer Communications INFOCOM, vol. 2, 2000, pp. 775-784.

[3] T. Deasy and W. Scanlon, "Simulation or measurement: The effect of radio map creation on indoor WLAN-Based localisation accuracy," Wireless Personal Communications, vol. 42, no. 4, pp. 563-573, 2007.

[4] A. Hatami and K. Pahlavan, "Comparative statistical analysis of indoor positioning using empirical data and indoor radio channel models," in 3rd IEEE Consumer Communications and Networking Conference (CCNC), vol. 2. IEEE, 2006, pp. 1018-1022.

[5] K. El-Kafrawy, M. Youssef, A. El-Keyi, and A. Naguib, "Propagation modeling for accurate indoor WLAN RSS-based localization," in IEEE Vehicular Technology Conference Fall (VTC2010-Fall), 2010, pp. 1-5.

[6] A. Haeberlen, E. Flannery, A. M. Ladd, A. Rudys, D. S. Wallach, and L. E. Kavraki, "Practical robust localization over large-scale 802.11 wireless networks," in 10th international conference on Mobile computing and networking (MobiCom), 2004, pp. 70-84.

[7] J. Park, D. Curtis, S. Teller, J. Ledlie et al., "Implications of device diversity for organic localization," in IEEE International Conference on Computer Communications INFOCOM, 2011, pp. 3182-3190.

[8] M. B. Kjærgaard, "Indoor location fingerprinting with heterogeneous clients," Pervasive and Mobile Computing, vol. 7, no. 1, pp. 31-43, 2011.

[9] J. Jemai, R. Piesiewicz, and T. Kurner, "Calibration of an indoor radio propagation prediction model at $2.4 \mathrm{GHz}$ by measurements of the IEEE 802.11b preamble," in IEEE 61st Vehicular Technology Conference (VTC 2005-Spring), vol. 1, 2005, pp. 111-115.

[10] S. Stavrou and S. Saunders, "Review of constitutive parameters of building materials," in Antennas and Propagation, 2003.(ICAP 2003). Twelfth International Conference on (Conf. Publ. No. 491), vol. 1. IET, 2003, pp. 211-215.

[11] A. Eleryan, M. Elsabagh, and M. Youssef, "AROMA: Automatic generation of radio maps for localization systems," CoRR, vol. abs/1002.1834, 2010.

[12] B. Li, J. Salter, A. Dempster, and C. Rizos, "Indoor positioning techniques based on wireless LAN," in 1st IEEE International Conference on Wireless Broadband and Ultra Wideband Communications, 2006, pp. 13-16.

[13] T. Roos, P. Myllymaki, H. Tirri, P. Misikangas, and J. Sievanen, "A probabilistic approach to WLAN user location estimation," International Journal of Wireless Information Networks, vol. 9, no. 3, pp. 155-164, Jul. 2002. 